\title{
Una propuesta de modelo educativo para las organizaciones exponenciales
}

\author{
José Antonio Álvarez López \\ Doctorando en la Universidad Rey Juan Carlos \\ joseantonio.alvarez@urjc.es \\ Ricardo Sampablo Buezas \\ Profesor del Centro de Estudios Financieros, CEF.- y de la \\ Universidad a Distancia de Madrid, UDIMA \\ ricardo.sampablo@udima.es
}

\section{Extracto}

La sociedad, las empresas y los profesionales actuales se encuentran en un complejo proceso de transformación, con la necesidad de incorporarse a un nuevo contexto digital empresarial, inherente a la transformación digital de las organizaciones, que se ha dado en llamar «nueva economía». En este contexto, los referentes son las organizaciones exponenciales, que incluyen competencias adicionales específicas. Con el fin de dar respuesta a este tipo de organizaciones, se propone un nuevo marco formativo, caracterizado por la capacitación tecnológica, que además evolucionará en paralelo con la transformación digital educativa, social, política, económica y empresarial.

En este trabajo de investigación se propone un nuevo marco formativo, basado en la adquisición de las competencias inherentes al liderazgo disruptor, que mejora la competitividad de los profesionales y, por ende, de las empresas.

Palabras clave: transformación digital; nueva economía; innovación disruptiva; organizaciones exponenciales; liderazgo disruptor. 


\title{
A proposal for an educational model for exponential organizations
}

\author{
José Antonio Álvarez López \\ Ricardo Sampablo Buezas
}

\section{Abstract}

Today's society, companies and professionals are in a complex process of transformation, with the need to join a new digital business context, inherent in the digital transformation of organizations, which has become known as the «new economy». In this context, the referents are exponential organizations, which include specific additional competences. In order to respond to this type of organization, a new training framework is proposed, characterized by technological training, which will also evolve in parallel with the digital educational, social, political, economic and business transformation.

In this research work, a new training framework is proposed, based on the acquisition of the skills inherent in disruptive leadership, which improves the competitiveness of professionals and, therefore, of companies.

Keywords: digital transformation; new economy; disruptive innovation; exponential organizations; disruptive leadership.

Citation: Álvarez López, J. A. and Sampablo Buezas, R. (2020). A proposal for an educational model for exponential organizations. Tecnología, Ciencia y Educación, 17, 149-179. 


\section{Sumario}

1. Introducción

2. Las organizaciones exponenciales

2.1. Contexto, definición y crecimiento exponencial

2.2. Modelos de negocio exponenciales

2.3. Características de las organizaciones exponenciales

3. Las tecnologías exponenciales

4. El capital humano exponencial

5. El modelo educativo inherente a las organizaciones exponenciales

5.1. Taxonomía competencial asociada al liderazgo disruptor

6. Propuesta para construir una acción formativa exponencial

6.1. El ecosistema de aprendizaje

6.2. Objetivos de aprendizaje

6.3. Características del diseño instruccional

7. Conclusiones

7.1. Futuras líneas de investigación

7.2. Conclusiones principales

Referencias bibliográficas 


\section{Introducción}

Según Howard Gardner, investigador en la Universidad de Harvard y Premio Príncipe de Asturias de Ciencias Sociales en 2011 (Gardner, 2012):

[...] el objetivo final de la educación es una ciudadanía educada que comprenda el mundo físico, el mundo biológico y el mundo social, y que sepa aplicar esta comprensión a su mundo personal, social y cultural [...] la educación ayudará a las generaciones más jóvenes a aceptar los desafíos del futuro, conservando al mismo tiempo las metas tradicionales de una educación (p. 316).

Luego, los fines de la educación están relacionados con la «comprensión del mundo» y con el hecho de «aceptar los desafíos del futuro». Es en estos dos aspectos donde resulta relevante, por un lado, comprender adecuadamente el mundo actual y, por otro, intentar vislumbrar los desafíos que se encontrarán los jóvenes en el futuro, aun siendo estos inciertos.

Con respecto a los dos aspectos mencionados, el análisis pasa indudablemente por el profundo cambio social que se ha producido con la irrupción de la tecnología de la información y la comunicación (TIC) en todos los ámbitos dentro del contexto de la cuarta revolución

Las empresas que mejor representan los nuevos modelos de negocio son las que Salim Ismail denominó como «organizaciones exponenciales» industrial, de la nueva economía, y, por ende, de los nuevos modelos de negocio propiciados por la innovación disruptiva (Bower y Christensen, 1995). Las empresas que mejor representan estos nuevos modelos, por otro lado tan exitosos, son las que Salim Ismail denominó como «organizaciones exponenciales» (Ismail, Malone y Van Geest, 2014).

Con el objetivo de dar respuesta a estos nuevos modelos de organización, y a la sociedad de la información y comunicación, el modelo educativo actual debe evolucionar. En esta investigación, se propone un nuevo marco formativo, aplicable en un contexto de organización exponencial, asociado al liderazgo disruptor, siendo esta la característica más significativa relacionada con la innovación disruptiva.

Para cumplir con este objetivo, se propone una nueva taxonomía competencial y un sistema educativo de aprendizaje invertido, centrado en el alumno, que mejora la competitividad de los profesionales y, por ende, de las empresas. 


\section{Las organizaciones exponenciales}

\subsection{Contexto, definición y crecimiento exponencial}

Cuando nos referimos a contextos de innovación disruptiva, es una evidencia que hay organizaciones que han conseguido rendimientos y crecimientos muy superiores a los de sus competidores (Kim y Mauborgne, 2014) y en tiempos mucho más reducidos (Mayer, 2016). Si se revisa el cuadro 1 , se puede comprobar que 7 de las 10 empresas más grandes del mundo en el año 2019 tienen menos de 50 años; 5, apenas 25 años o menos; e incluso una de ellas, Facebook, ha tardado solo 15 años en posicionarse la sexta del mundo. No es casualidad tampoco que 7 de las 10 sean empresas tecnológicas.

Cuadro 1. Ranking mundial de las 10 mayores empresas por capitalización y fecha de fundación (2019)

\begin{tabular}{|c|l|l|}
\hline $\begin{array}{c}\text { Puesto en el } \\
\text { ranking mundial }\end{array}$ & \multicolumn{1}{c|}{ Compañía } & Fecha de fundación \\
\hline 1 & Apple & 1 de abril de 1976 \\
\hline 2 & Microsoft & 4 de abril de 1975 \\
\hline 4 & Amazon & 5 de julio de 1994 \\
\hline 5 & Alphabet & 2 de octubre de $2015^{*}$ \\
\hline 6 & Berkshire Hathaway & 1839 \\
\hline 7 & Facebook & 4 de febrero de 2004 \\
\hline 8 & Alibaba & 4 de abril de 1999 \\
\hline 9 & Tencent Holdings & 11 de noviembre de 1998 \\
\hline 10 & JP Morgan Chase & 1799 \\
\hline
\end{tabular}

* Alphabet procede de Google, que fue fundada el 4 de septiembre de 1998. 
En el contexto descrito, de organizaciones que se apoyan en "tecnologías exponenciales", lo que quiere decir que cada año multiplican su potencia de un modo exponencial, se obtienen, además, crecimientos exponenciales (véase figura 1). Estos crecimientos se diferencian de los lineales en la aceleración del crecimiento, destacándose la importancia de la «ley de rendimientos acelerados» de Ray Kurzweil, que es una extensión de la «ley de Moore» (Bloomberg, 2015; Kurzweil, 2004).

Figura 1. Crecimiento lineal versus exponencial

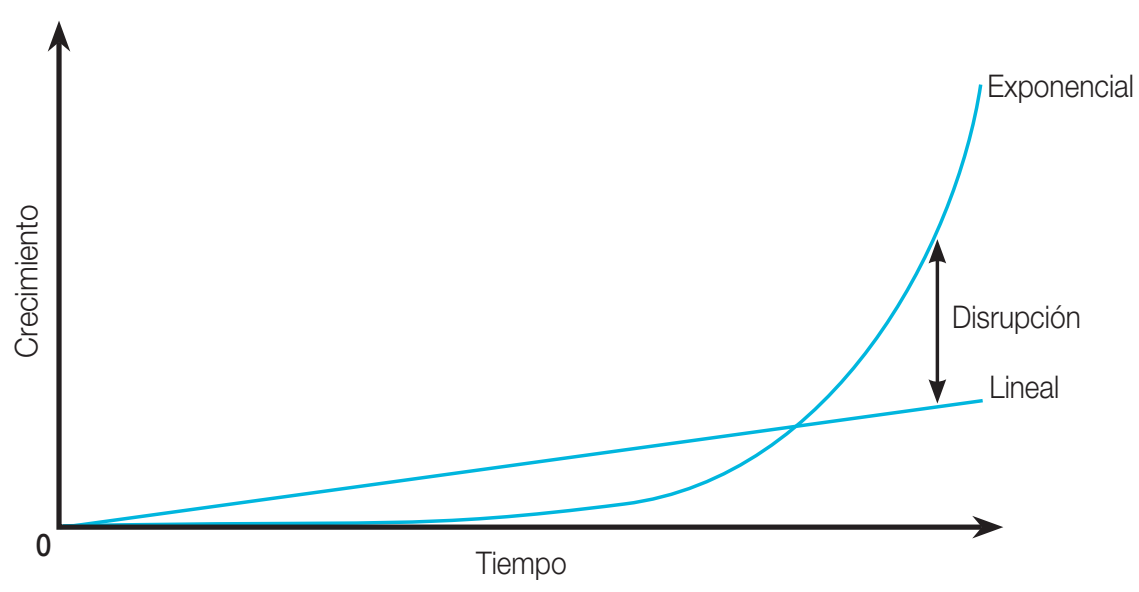

Fuente: Ismail et al. (2016).

Salim Ismail, en su libro Exponential Organizations: Why New Organizations Are Ten Times Better, Faster, and Cheaper Than Yours (and What to Do about It) (Ismail et al., 2016), definió las "organizaciones exponenciales» como "aquellas cuyo impacto o resultado es desproporcionadamente grande -al menos 10 veces superior- al compararlas con sus iguales, gracias al uso de nuevas técnicas organizativas que se sirven de las tecnologías aceleradoras».

Algunas de estas organizaciones se diseñaron apoyándose en la idea de "abundancia» que plasmó Peter Diamandis en su libro The Road to Abundance-Innovation, Disruption, and Opportunity: «Conspirando para resolver los mayores problemas del mundo» (Diamandis, 2016).

Todas estas observaciones, el ritmo constante, extraordinario y aparentemente imposible de crecimiento, llevaron a Kurzweil (2004) a plantear los siguientes comentarios al respecto de estas organizaciones:

- Se construyen sobre tecnologías de la información que toman lo que una vez fue físico en la naturaleza y lo desmaterializan en el mundo digital a demanda. Esto 
ha sucedido con multitud de sectores, como, por ejemplo, la banca, el comercio, que ahora es electrónico, la educación, etc.

- El patrón de duplicación acelerado de crecimiento se alimenta de la información. Una vez que cualquier ámbito, disciplina, tecnología o industria tiene acceso y se alimenta de información, su precio/rendimiento comienza a duplicarse más o menos anualmente.

- Una vez que los patrones de duplicación comienzan no se interrumpen.

- Existen tecnologías clave de hoy en día que tienen acceso a la información y están siguiendo la misma trayectoria; por ejemplo, la inteligencia artificial.

Estos modelos se deben construir de forma que su desarrollo sea sostenible (Aguinis y O'Boyle, 2014; Birkin, Polesie y Lewis, 2009; Briggs y Shingles, 2015; Farahani, Tourang, Yousefpour, Naraghi y Javadian, 2012; Lee, Nunes, Canning, Meyer y Stanley, 1998; Zazzerini, 2016).

Estas organizaciones no solo necesitan de tecnologías exponenciales, sino que se apoyan, además, de un segundo ingrediente: metodologías y modelos de innovación disruptiva.

Por consiguiente, el reto real que se plantea no será predecir las implicaciones de las tecnologías exponenciales, o descubrir su potencial de disrupción, sino crear los nuevos tipos de organizaciones y modelos de negocio que aprovechen las posibilidades tecnológicas que tenemos hoy en día (Ismail et al., 2014; Palao Gil, 2016) y que consigan crear crecimientos exponenciales, explotando, por ejemplo, varias vías de mejora continua simultáneamente (Pitcher, 2017). De esta manera, no solo se conseguirá sobrevivir a los cambios actuales del entorno, sino que también obtendremos resultados muy superiores.

Una vez que se ha contextualizado el concepto de «exponencial» en el sentido de las empresas que consiguen rendimientos muy superiores a los habituales, en menos tiempo, conviene conocer con detalle los modelos de negocio que definen este tipo de organizaciones. El tema se trata en el siguiente apartado.

\subsection{Modelos de negocio exponenciales}

Tal y como nos describe el principal think tank del mundo sobre el crecimiento exponencial, la Singularity University (Solomon y Pijl, 2017), un modelo de negocio define cómo se crea y se entrega el valor en una empresa. Es una forma organizada de establecer suposiciones sobre recursos clave, socios y actividades de su cadena de valor. Estos incluyen su propuesta de valor, las relaciones con los clientes, los canales y segmentos de clientes, las estructuras de costes y los flujos de ingresos. Un modelo de negocio exponencial analiza las mismas áreas clave que un modelo de negocio tradicional, pero tiene objetivos radicalmente diferentes. 
La mayoría de los modelos de negocio son lineales, diseñados para aumentar las ganancias o disminuir los costes en un $10 \%$. Con un modelo de negocio exponencial, pensamos en términos de cambios que son 10 veces mayores o menores que el valor actual; la abreviatura común para este objetivo es simplemente «10X». Los modelos de negocio de las organizaciones exponenciales se apoyan en metodologías específicas, como la agile exponential software (Dubinsky y Hazzan, 2019), o la introducida por Osterwalder y Pigneur (2010) en su libro Business Model Generation: A Handbook for Visionaries, Game Changers, and Challengers. Este esquema resume los elementos centrales de un modelo de negocio en nueve bloques, que Solomon y Pijl (2017) consideran como los nueve principios de diseño para la transformación exponencial (véase figura 2). Modelo adaptado también por Sánchez (2020).

Figura 2. Nueve principios de diseño para la transformación exponencial

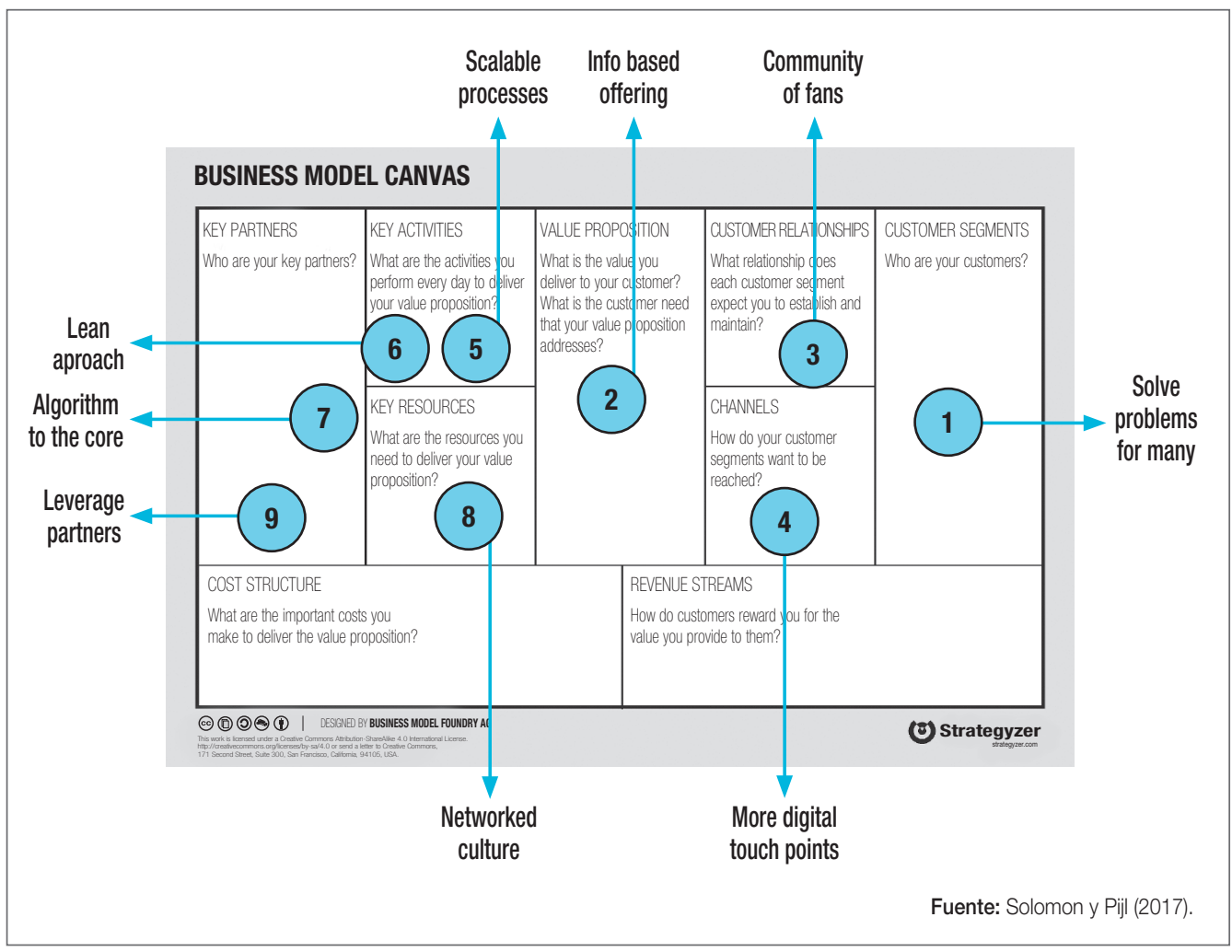

Los nueve principios de diseño para la transformación exponencial se describen a continuación:

- Segmentos de clientes. Se intenta resolver un problema para las masas. La tecnología permite a las organizaciones llegar a mercados completamente nuevos de 
manera masiva y viral. A medida que la población mundial se acerca a los 7.500 millones, las empresas y organizaciones con modelos de negocios exponenciales pueden ayudar a cerrar la brecha entre nuestra creciente población y los recursos que necesitan. Muchas compañías comienzan con una oferta central a los clientes para satisfacer una necesidad, como Uber y el transporte personal, y luego amplían sus servicios para satisfacer otras necesidades, como Uber Eats o Uber Health.

- Propuesta de valor: servicios y plataformas basados en información. A medida que las empresas digitalizan sus productos y servicios, no solo crean nuevas versiones de sus ofertas tradicionales, sino que también crean mercados completamente nuevos. La plataforma de Airbnb vuelve a imaginar alojamientos a corto plazo; Slack digitaliza la colaboración y el intercambio de conocimientos; la firma de genética de consumo 23andMe ofrece una secuencia de ADN (ácido desoxirribonucleico) asequible para cualquier persona, etc. Cada negocio, independientemente de la industria, explora cómo y qué digitalizar en su propuesta de valor existente para no solo servir mejor a los clientes existentes, sino también para abrir potencialmente nuevos intercambios de valor.

- Relaciones: construir una comunidad de fanáticos. Cuando se desea trabajar hacia una solución 10X, se debe integrar a los clientes en una base de admiradores y colaborar con ellos posteriormente. La personalización habilitada por el usuario de funciones básicas, como los filtros en Instagram o las lentes aumentadas en Snap, permite a los usuarios crear algo único y compartir sus creaciones en múltiples puntos de contacto, actuando como una distribución viral y a modo de marketing de canal.

- Canales: multimodales y sociales. Muchas empresas exponenciales están utilizando la colaboración social para conectar medios físicos y digitales múltiples para mejorar el valor de su oferta principal. Piénsese en esto como contenido generado por el usuario amplificado a través de las externalidades de la red: cuantas más personas contribuyan a la plataforma, más valioso será el servicio. Las empresas de más rápido crecimiento utilizan esta estrategia como motor de adquisición de clientes, compromiso y valor de por vida. La aplicación de tráfico Waze combinó los datos GPS con la entrada de tráfico en tiempo real de los usuarios utilizando métodos de gamificación para hacer que el proceso sea divertido y atractivo. Waze fue comprado por Google por 1.300 millones de dólares cuando solo tenían 100 empleados y, desde entonces, se ha convertido en el servicio de referencia para los viajeros.

- Actividades clave: procesos automatizados y escalables. La tecnología puede ayudar a analizar y automatizar rutinas para disrumpir los métodos tradicionales de fabricación o entrega. Amazon ha usado robots durante mucho tiempo para almacenar y recuperar productos de sus almacenes y se están haciendo pruebas para realizar las entregas con drones. También se dio cuenta de que una de sus actividades estratégicas más importantes, el almacenamiento en la nube y de datos, podría convertirse en un recurso valioso para otros. Amazon Web Services (AWS), un servicio de alquiler de infraestructura informática, fue lanzado en 2006. Diez años después, contribuyó con el $56 \%$ del crecimiento de Amazon y se espera que, en menos de cinco años, sea un negocio de 100.000 millones de dólares. 
- Actividades clave: enfoque lean. ¿Por qué General Electric se está volviendo más exponencial? Utilizan un enfoque lean para todas las funciones básicas de su negocio, enfatizando ciclos rápidos de experimentación y aprendizaje. Al establecer procesos lean en todas sus funciones, permite que los trabajadores asuman riesgos y obtiene datos sobre su negocio de los que aprender.

- Actividades clave, recursos y socios: algoritmo para el core. Google es uno de los mejores ejemplos de una empresa basada en un algoritmo (para clasificar sitios web), que luego se mejora mediante el aprendizaje automático.

- Recursos clave: cultura en red. Los empleados deben trabajar en forma de redes para socializar y compartir ideas y experiencias en tiempo real. Dar a los empleados autonomía para hacer lo que necesitan en una cultura de apoyo y abierta descentraliza y acelera la toma de decisiones, abriendo la posibilidad de un modelo de negocio 10X. Zappos ha sido uno de los pioneros en construir una cultura de «holocracia», eliminando puestos de gestión profesional y empoderando a los trabajadores sin burocracia adicional. Las compañías como Microsoft apuestan mucho por el software social para apoyar los esfuerzos espontáneos de colaboración, incorporando redes y funcionalidad social en todas sus nuevas suites de ofimática.

- Socios: construir relaciones poco comunes. Muchos modelos de negocio exponenciales provienen de «socios poco comunes»: distintos tipos de empresas de diferentes industrias que trabajan juntas para beneficiarse del valor integrado. La compañía de drones Matternet y Mercedes-Benz, recientemente, unieron fuerzas para crear una solución de entrega integrada, diseñada para transformar la forma en que las personas reciben productos ligeros bajo pedido.

De todos estos principios de diseño, el último es el más poderoso. El hecho de decidir el nivel de integración de la organización plantea nuevas situaciones que difieren de las organizaciones tradicionales. Esta cuestión se aplica a todos los activos intensivos en capital, incluidos los equipos, el talento o las nuevas ideas innovadoras. Los siguientes aspectos que se van a tratar, una vez analizados los modelos de negocio exponenciales, son características que necesitan las organizaciones para cumplir con los requisitos de los modelos expuestos. Estas características se desarrollan a continuación.

\subsection{Características de las organizaciones exponenciales}

De igual modo que se encuentran similitudes en la forma acelerada en la que las organizaciones exponenciales crecen, la diversidad de compañías, sectores y formas de llegar a ese crecimiento complica la estandarización de los factores que están detrás de ese crecimiento; no obstante, los autores del libro Exponential Organizations... (Ismail et al., 2016) han analizado las 100 startups que han experimentado el mayor crecimiento mundial desde el 2010 al 2016. Como resultado de estos trabajos, se han identificado ciertos rasgos comunes que estaban presentes en todas las organizaciones exponenciales. Estos rasgos incluyen un propósito de transformación masivo (PTM), entre una decena de atributos que reflejan los mecanismos 
internos y externos que están sirviendo para conseguir ese crecimiento exponencial. Se utiliza el acrónimo SCALE ${ }^{1}$ para reflejar los cinco atributos externos e IDEAS ${ }^{2}$ para los cinco atributos internos. No todas las organizaciones exponenciales presentan los 10 atributos, pero, cuantos más reúnan, más escalables suelen ser. Los autores del trabajo indican que tener un mínimo de cuatro atributos implementados convierte a la compañía en organización exponencial y hace que se acelere su crecimiento, superando a sus competidores.
Como se puede ver en la figura 3, donde se representan gráficamente los atributos de las organizaciones exponenciales, el hemisferio derecho simboliza el crecimiento, la creatividad y la incertidumbre, mientras que el hemisferio izquierdo se ocupa del orden, el control y la estabilidad

Los atributos se muestran de forma gráfica a continuación (véase figura 3). El hemisferio derecho simboliza el crecimiento, la creatividad y la incertidumbre, mientras que el hemisferio izquierdo se ocupa del orden, el control y la estabilidad.

Figura 3. Atributos de las organizaciones exponenciales

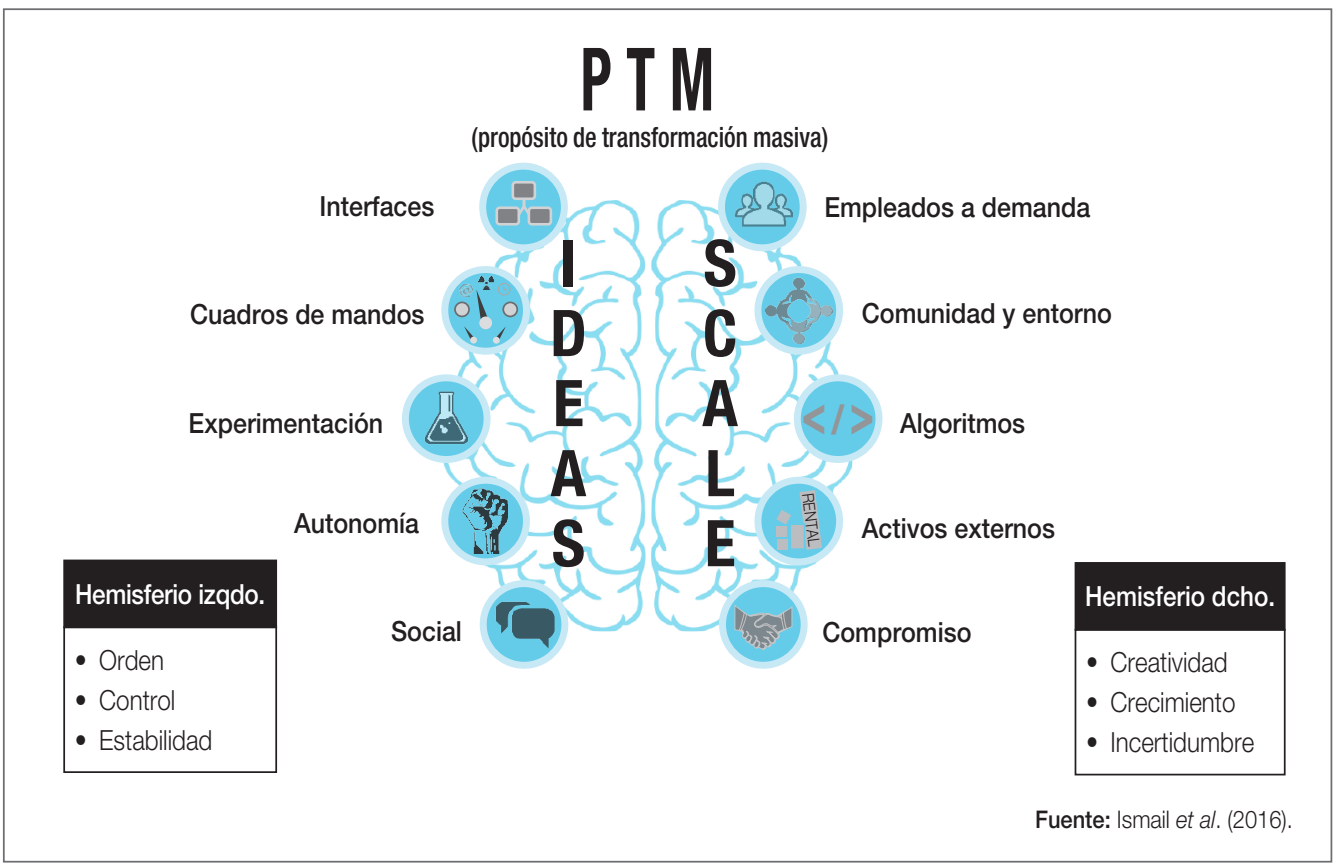

\footnotetext{
1 Véase la explicación del acrónimo SCALE en el cuadro 2.

2 Véase la explicación del acrónimo IDEAS en el cuadro 3.
} 
EI PTM es el propósito mayor al que aspira la organización. Todas las organizaciones exponenciales tienen uno y, en ocasiones, dado su alcance, puede ser milagroso. Encontramos el ejemplo siguiente en Google, cuyo PTM es «organizar la información mundial». Fácil de entender en los días actuales, pero no evidente en el momento en el que se lo plantearon. Esto no se debe confundir con la misión de la compañía. EI PTM es capaz de generar un movimiento cultural alrededor de la idea y, por lo tanto, debe ser inspirador. EI PTM resulta ser una ventaja competitiva respecto a los competidores. Está muy ligado a los pioneros en un nuevo sector.

A continuación, en el cuadro 2, se presentan los atributos asociados al modelo SCALE y, en el cuadro 3 , los atributos asociados al modelo IDEAS.

\section{Cuadro 2. Atributos asociados al modelo SCALE}

\begin{tabular}{l|l}
\multicolumn{1}{c|}{ Atributo SCALE } & \multicolumn{1}{c}{ Descripción } \\
\hline $\begin{array}{l}\text { S (staff on demand/empleados a de- } \\
\text { manda). }\end{array}$ & $\begin{array}{l}\text { Contratación de servicios o personal interno o externo, depen- } \\
\text { diendo de los objetivos que haya que cumplir, flexibilizando las } \\
\text { relaciones laborales y mercantiles. }\end{array}$ \\
$\begin{array}{ll}\text { C (community and crowd/comunidad } \\
\text { y entorno). }\end{array}$ & $\begin{array}{l}\text { Generación de comunidad, interaccionando y generando ideas } \\
\text { dentro y fuera de la propia organización. }\end{array}$ \\
\hline A (algorithms/algoritmos). & $\begin{array}{l}\text { Desarrollo de métricas y algoritmos que permitan gestionar y } \\
\text { utilizar adecuadamente la información convirtiéndola en un fac- } \\
\text { tor de evolución. }\end{array}$ \\
\hline (leveraged assets/activos externos). & Utilización de activos sin necesidad de propiedad. \\
\hline E (engagement/compromiso). & Generación de compromiso en la red, vinculado con la organi- \\
& zación y el PTM. \\
\hline
\end{tabular}

Fuente: elaboración propia a partir de Ismail et al. (2016).

\section{Cuadro 3. Atributos asociados al modelo IDEAS}

\section{Atributo IDEAS}

I (interfaces/interfaces).

\section{Descripción}

Son procesos de filtrado y de unión con los que las organizaciones exponenciales construyen puentes entre las externalidades SCALE y los marcos de control internos de IDEAS. 


\begin{tabular}{|l|l|}
\hline \multicolumn{1}{|c|}{ Atributo IDEAS } & \multicolumn{1}{c}{ Descripción } \\
\hline D (dashboards/cuadros de mando). & $\begin{array}{l}\text { Maneras nuevas de medir, a tiempo real, de forma adaptable, } \\
\text { que incluya todas las métricas sobre la compañía y los emplea- } \\
\text { dos y accesible por todos. }\end{array}$ \\
\hline E (experimentation/experimentación). & $\begin{array}{l}\text { Implementación de la tecnología lean startup para testar hipó- } \\
\text { tesis y experimentar constantemente con riesgos controlados. }\end{array}$ \\
\hline A (autonomy/autonomía). & $\begin{array}{l}\text { Equipos multidisciplinares que se autoorganizan y que operan } \\
\text { con autoridad descentralizada. }\end{array}$ \\
\hline $\begin{array}{l}\text { S (social technologies/tecnologías so- } \\
\text { Generación de interacciones no formales entre los miembros de } \\
\text { la red. Siete elementos clave: objetos sociales, corrientes de acti- } \\
\text { vidad, gestión de tareas, compartir archivos, telepresencia, mun- } \\
\text { dos virtuales y detección emocional. }\end{array}$ \\
\hline
\end{tabular}

Fuente: elaboración propia a partir de Ismail et al. (2016).

Las organizaciones se adaptan a estos modelos exponenciales, haciéndose cada vez más escalables (Mohout, 2017), incorporando lo que se ha dado en llamar «líderes disruptores», que sí son capaces de dirigir a los equipos hacia estas nuevas formas de organización.

Una vez caracterizado el modelo de las organizaciones que, previsiblemente, establecerán el camino que hay que seguir, resulta relevante establecer las tecnologías exponenciales y/o disruptivas que serán utilizadas por estas nuevas empresas. Se detallan a continuación.

\section{Las tecnologías exponenciales}

En los análisis del Global McKinsey Institute, incluidos en su informe Disruptive Technologies: Advances that Will Transform Life, Business, and the Global Economy (Manyika et al., 2013), se tienen en cuenta las tecnologías en rápida evolución y potencialmente transformadoras en el futuro, que abarcan las tecnologías de la información, las ciencias biológicas, la ciencia de los materiales, la energía y otros campos.

En el mencionado informe de McKinsey, se propone identificar cuáles de estas tecnologías podrían tener un impacto masivo y económicamente disruptivo hasta el año 2025. También trata de entender cómo estas tecnologías podrían cambiar nuestro mundo y cómo deberían responder a estos cambios los líderes de las empresas y otras instituciones. 
Un análisis estructurado para clasificar las tecnologías con el potencial de transformarse y «disrumpir» en la próxima década o dos nos permitirá evaluar el impacto potencial basado en lo que podemos saber en la actualidad y poner estas tecnologías en una perspectiva con potencial útil. El objetivo es identificar las tecnologías que serán líderes en el año 2025, que pueden aparecer desde cualquier campo o surgir de cualquier disciplina científica. Todas ellas comparten cuatro características:

- Alta tasa de cambio tecnológico. La tecnología progresa rápidamente y experimenta avances importantes. Las tecnologías disruptivas generalmente muestran una rápida tasa de cambio en capacidades, en rendimiento/precios, en relación con los bienes sustitutivos o los enfoques alternativos, y experimentan progresos que impulsan tasas de cambio aceleradas o mejoras discontinuas de la capacidad.

- Amplio alcance del impacto potencial. El alcance potencial del impacto es amplio. Para ser económicamente disruptiva, una tecnología debe tener un amplio alcance: impactar en empresas e industrias y afectar (o dar lugar a) una amplia gama de máquinas, productos o servicios.

- Posibilidad de impacto económico masivo. Una tecnología económicamente disruptiva debe tener el potencial de crear un impacto económico masivo. El valor en juego debe ser grande, en términos de agentes que podrían verse afectados, de incrementos del producto interior bruto (PIB) que podrían obtenerse y de formación de capital fijo que podría quedar obsoleto (Bughin et al., 2016).

- Potencial significativo de impacto económico disruptivo. Las tecnologías importantes tienen el potencial de cambiar drásticamente el statu quo. Pueden transformar el modo en el que las personas viven y trabajan. Como crean nuevas oportunidades, intercambian sus combinaciones de ocio/trabajo con las empresas, impulsan el crecimiento o cambian las ventajas competitivas de los países.

En el citado informe de McKinsey (Manyika et al, 2013), se analizan más de 100 tecnologías distintas, reduciendo la enorme lista a 12.

Estas tecnologías elegidas son las que se identifican con el potencial de afectar a miles de millones de consumidores, cientos de millones de trabajadores y billones de dólares de actividad económica en todas las industrias.

Estas tecnologías se enumeran y describen en el cuadro 4, incluyendo, de esta manera, las que pueden tener mayor impacto. 


\section{Cuadro 4. Las 12 tecnologías con potencial para ser económicamente disruptivas}

\section{Tecnología}

Internet móvil.

Automatización del trabajo del conocimiento.

\section{Descripción}

Dispositivos móviles cada vez más capaces y conectividad a internet cada vez más económica.

Sistemas de software inteligentes que pueden realizar tareas de trabajo de conocimiento que incluyen comandos no estructurados y juicios sutiles.

Internet de las cosas.

Redes de bajo precio de sensores y para la recogida de datos, monitorización, toma de decisiones y de procesos.

Tecnología en la nube (cloud).

Uso de optimización de recursos de hardware y software de computación desde una red o internet, como un servicio.

Robótica avanzada.

Robots cada vez más capaces, con sentidos, destreza e inteligencia mejorados, utilizados para automatizar tareas o mejorar a los humanos.

Vehículos autónomos y casi autónomos.

Vehículos que pueden navegar y operar con intervención humana reducida o nula.

Ciencia genómica de próxima generación.

Secuenciación génica rápida y de bajo coste, análisis avanzado con big data y biología sintética ("escritura" de ADN).

Almacenamiento de energía.

Dispositivos o sistemas que almacenan energía para su uso posterior, incluidas las baterías.

Impresión 3D.

Técnicas de fabricación aditiva para crear objetos mediante la impresión de capas de material basadas en modelos digitales.

Materiales avanzados.

Materiales diseñados para tener características adicionales (por ejemplo, resistencia, peso, conductividad) o funcionalidad.

Exploración y recuperación avanzadas de petróleo y gas.

Energías renovables.
Técnicas de exploración y recuperación que hagan económica la extracción de petróleo y gas no convencional.

Generación de electricidad a partir de fuentes renovables con un impacto climático perjudicial reducido.

Fuente: traducción a partir de Manyika et al. (2013). 
En las 12 tecnologías descritas no están incluidas todas las que tienen potencial para ser económicamente disruptivas en 2025. Aunque muchas otras tecnologías avanzadas no tienen el mismo potencial de impacto económico para 2025, merecen también ser vigiladas y consideradas en opinión de los autores del informe. No obstante, esto podría cambiar si se diesen avances repentinos u otros factores, como un mayor apoyo de políticas públicas.

Para describir la productividad y el crecimiento que tendrán estas tecnologías, hay que mencionar uno de los impulsores clave: la TIC. La innovación en la industria es más rápida, más compleja y está más interconectada que nunca. Las empresas que no puedan adaptarse a los cambios que incorporan las infraestructuras comunes de telecomunicaciones no podrán sobrevivir en las próximas décadas. La movilidad, el ancho de banda, el desarrollo de las plataformas y metadatos están dando forma a la empresa a través de la innovación. Desde las nuevas empresas hasta las corporaciones más arraigadas, la TIC, sus innovaciones y sus avances están cambiando la economía. La difusión generalizada de esta tecnología a través de la industria y sus sectores garantiza que ningún aspecto de la nueva economía pueda permanecer intacto sin evolucionar (Australian Government, 2013). Un aspecto del panorama tecnológico cambiante es la digitalización: la adopción generalizada de servicios digitales conectados por parte de consumidores, empresas y Gobiernos.

Varios autores coinciden en señalar que las tecnologías asociadas con la Industria 4.0 están, por lo tanto, sujetas a la integración con las políticas y estrategias de la Unión Europea (Comisión Europea, 2015; Negreiro y Madiega, 2019; Parlamento Europeo, 2019).

En el estudio de tendencias propuesto por Gartner para el año 2020, Gartner Top 10 Strategic Technology Trends for 2020 (Burke et al., 2019; Panetta, 2019), se presenta una evolución del foco de las tendencias hacia la estructuración en torno a la idea de «espacios inteligentes centrados en las personas», lo que significa considerar cómo estas tecnologías afectarán a las personas (es decir, clientes, empleados, etc.) y a los lugares en los que viven (es decir, hogar, oficina, etc.).

Tal y como aseveró Brian Burke (Burke et al., 2019), vicepresidente de investigación de Gartner, «estas tendencias tienen un profundo impacto en las personas y en los espacios que habitan».

Estas tendencias no pueden considerarse de manera aislada. Son los líderes en TIC quienes deben decidir qué combinación de tendencias impulsará la mayor innovación.

Las tendencias identificadas por Gartner para 2020 son las siguientes:

- Hiperautomatización. Así como la automatización utiliza la tecnología para automatizar tareas que alguna vez requirieron de humanos, la hiperautomatización se ocupa de la aplicación de tecnologías avanzadas, incluida la inteligencia artificial y el aprendizaje automático (machine learning), para automatizar cada vez más los 
procesos. La hiperautomatización se extiende a través de una gama de herramientas que pueden automatizarse, pero también se refiere a la sofisticación de la automatización (es decir, descubrir, analizar, diseñar, automatizar, medir, monitorear y reevaluar). Como ninguna herramienta única puede reemplazar a los humanos, la hiperautomatización, hoy en día, implica una combinación de herramientas, incluida la automatización de procesos robóticos (robotic process automation), el software inteligente de gestión empresarial (intelligent business management software) y la inteligencia artificial con el objetivo de tomar decisiones cada vez más basadas en sus propias decisiones (Norlean, 2019).

Aunque no es el objetivo principal de la hiperautomatización, a menudo, da como resultado la construcción de un gemelo digital de la organización (digital twin of the organization), lo que permite visualizar cómo interactúan las funciones, los procesos y los indicadores clave de rendimiento para generar valor. Luego, el digital twin of the organization se convierte en una parte integral del proceso de hiperautomatización, brindando inteligencia continua y en tiempo real sobre la organización e impulsando oportunidades comerciales significativas.

- Multiexperiencia. Este punto se centra en la forma en que las personas interaccionan, perciben y controlan el mundo digital y las aplicaciones relacionadas. En esta tendencia, la idea tradicional de un computador evoluciona desde un único punto de interacción para incluir interfaces multisensoriales y multitáctiles, como dispositivos portátiles y sensores informáticos avanzados.

En el futuro, esta tendencia, previsiblemente, se convertirá en lo que se llama una "experiencia ambiental», pero, actualmente, la multiexperiencia se enfoca en experiencias inmersivas que usan interfaces de realidad aumentada, realidad virtual, realidad mixta, multicanal humano-máquina y tecnologías de detección. La combinación de estas tecnologías se puede utilizar para una superposición de realidad aumentada simple o para una experiencia de realidad virtual totalmente inmersiva.

- Democratización. La democratización de la tecnología significa proporcionar a las personas un acceso fácil a la experiencia técnica o comercial sin necesidad de una capacitación larga y costosa. Esta democratización se centra en cuatro áreas clave:

- Democratización de los datos y del análisis.

- Democratización del desarrollo.

- Democratización del diseño.

- Democratización del conocimiento.

- Humano «aumentado». Se refiere al uso de la tecnología para mejorar las experiencias físicas y cognitivas de las personas y es el resultado de la intersección de muchos sectores, como la multiexperiencia, la hiperautomatización, las cosas 
autónomas, etc. Este «aumento» cambia una capacidad física inherente al implantar o alojar una tecnología dentro o sobre el cuerpo de la persona. Por ejemplo, las industrias automotriz o minera usan dispositivos portátiles para mejorar la seguridad de los trabajadores. En otras industrias, como en el comercio minorista y los distribuidores de viajes, los dispositivos portátiles se utilizan para aumentar la productividad de los trabajadores.

- Transparencia y trazabilidad. La evolución de la tecnología está creando una crisis de confianza. Los consumidores son cada vez más conscientes del valor de la información personal y exigen que haya un control de cómo las organizaciones administran y usan esos datos. La transparencia y la trazabilidad son elementos críticos para apoyar estas necesidades de ética digital y privacidad.

- Edge computing. Es una tecnología donde el procesamiento de la información y la recopilación y entrega de contenido se colocan más cerca de las fuentes de información, de los repositorios y de los consumidores de dicha información. Una práctica que, además, hace que se reduzca la latencia (el tiempo que tarda en transmitirse un paquete dentro de la red, factor clave en las conexiones de internet). Esto incluye toda la tecnología en internet de las cosas (según Gartner, el edge computing se convertirá en un factor dominante en casi todas las industrias y casos de uso).

- Nube distribuida. Se refiere a la distribución de servicios de nube pública a ubicaciones fuera de los centros de datos físicos del proveedor de la nube, pero que aún están controlados por el proveedor. En la nube distribuida, el proveedor de la nube es responsable de todos los aspectos de la arquitectura del servicio en la nube, de la entrega, de las operaciones, del gobierno y de las actualizaciones. La evolución de la nube pública centralizada a la nube pública distribuida marca el comienzo de una nueva era de computación en la nube.

- Objetos autónomos. Se refiere a los drones, a los aviones no tripulados, a lo vehículos autónomos, a los barcos y otros dispositivos que explotan la inteligencia artificial para realizar tareas que generalmente llevan a cabo los humanos. Esta tecnología opera en un espectro de inteligencia que va desde semiautónomas hasta totalmente autónomas y en una variedad de entornos, incluidos el aire, el mar y la tierra. Si bien, actualmente, los objetos autónomos existen principalmente en entornos controlados, como en una mina o en un almacén, eventualmente, evolucionarán y se encontrarán en espacios públicos abiertos; además, aprovecharán la inteligencia artificial para comportarse de forma más avanzada, de modo que puedan interactuar de manera más natural con su entorno y con las personas.

- Cadena de bloques, también llamada «blockchain». A pesar de que a la tecnología de cadena de bloques le falta madurez para ser aplicada de forma intensiva en la empresa, su potencial disruptivo y la generación de ingresos que se prevé que genere hacen que las organizaciones deban comenzar a evaluar su uso o, al menos, empezar a experimentar con esta opción. 
La cadena de bloques podría remodelar industrias enteras y mejorar la confianza y la transparencia de los ecosistemas empresariales, al tiempo que reducir los costes y los tiempos de liquidación de las transacciones, así como optimizar el flujo de caja.

- Inteligencia artificial y seguridad. El auge de la inteligencia artificial (especialmente, las técnicas de machine learning) para mejorar la toma de decisiones humanas conlleva muchos beneficios, pero también implica importantes retos en materia de seguridad. Gartner prevé que aumenten los ataques con el auge del internet de las cosas, el cloud computing, los microservicios y los sistemas altamente conectados en espacios inteligentes. Por ello, la consultora recomienda a los responsables de seguridad y gestión de riesgos que centren su atención en la protección de los sistemas que funcionan con inteligencia artificial, que aprovechen esta última para mejorar la defensa de la seguridad y que anticipen el uso que puedan hacer de ella los atacantes para pertrechar sus actos.

Una vez revisadas las tecnologías que con alta probabilidad se convertirán en disruptivas, y en las que se apoyarán las organizaciones exponenciales, resulta relevante cuestionarse qué marcos formativos se aplicarán y qué competencias son las claves, para preparar a los alumnos en el contexto de la nueva economía. Se comenzará por definir el «capital humano exponencial».

\section{El capital humano exponencial}

A la vista de las singulares características que presentan las organizaciones exponenciales, en cuanto a sus modelos de negocio y de lo complejo y variado de las tecnologías exponenciales en las que se apoyan, tiene sentido reflexionar sobre las habilidades que el capital humano, alineado con estos contextos, necesitará incorporar. A continuación se desarrolla este concepto: el «capital humano exponencial».

Como dicen Martínez-Olvera, Esquivel-Gámez y Martínez (2015), en el escenario actual, dos factores destacan en la conceptualización de la sociedad del conocimiento: los procesos educativos y las TIC (Krüger, 2006). Las reflexiones anteriores arguyen la propagación de modelos tecnoeducativos, cuyo sustento pedagógico otorga plusvalía al uso e implementación de tecnología en la educación, haciendo factible la formación de comunidades de aprendizaje y la adquisición de competencias digitales, aspecto deseable en la formación del ciudadano del siglo XXI.

En esta línea, y desde una aproximación más reciente de la consultora BCG en Fixing the Global Skills Mismatch (Puckett et al., 2020), se considera que el capital humano está bajo una intensa presión en todo el mundo a medida que las poderosas fuerzas -la globalización, los cambios demográficos y regionales, la digitalización, la urbanización y las formas 
de empleos virtuales e informales- ganan impulso. Estas fuerzas están cambiando cómo, dónde y cuándo trabajan las personas, pero seguimos basando el desarrollo del capital humano en un modelo de mediados del siglo XX: educación estandarizada y un trabajo para toda la vida. Esto no puede funcionar en el mundo actual, que exige una competencia de crecimiento continuo. El cambio tecnológico continuo y las transformaciones del mercado requieren pensamiento flexible, aprendizaje rápido y continuo y movilidad. En lugar de la estandarización masiva, debemos adoptar la singularidad de la masa y la centralidad humana.

El capital humano no es una «mercancía homogénea», ya que incluye baby boomers, que nacieron durante los albores de la era espacial; la generación X, nacida en la ola de la informática personal; los millennials, que crecieron con los teléfonos móviles; y la generación Z, que son los conocidos como «nativos digitales». Cada grupo tiene intereses, valores, conocimientos, habilidades, experiencias y ambiciones distintivos. Para participar activamente en la sociedad y la economía, necesitan capacitación, empleos, carreras profesionales y nuevas formas de desarrollar su vida profesional.

Asimismo, Puckett et al. (2020) señala que la economía futura exige un nuevo enfoque. Para aprovechar todo el potencial del capital humano, debemos abordar (entre otros) los siguientes desafíos:

- Estamos insuficientemente enfocados en la capacitación para trabajos que aún no han aparecido. Se espera que el $27 \%$ de los trabajos disponibles en 2022 necesitarán de roles profesionales que todavía no existen.

- Una mayoría de la fuerza laboral no se involucra en el aprendizaje a lo largo de toda la vida, ni en el reciclaje continuo, pero, por otro lado, las habilidades se están volviendo cada vez más obsoletas, a un ritmo cada vez más rápido (las habilidades técnicas, por ejemplo, se desactualizarán entre 2 y 5 años). Estas circunstancias aumentan la necesidad de volver a capacitar y mejorar a los trabajadores.

A tenor de lo expuesto en el contexto formativo, se considera que el desarrollo efectivo de este modelo de competencias se basa, como principio general, en un proceso de «reinvención de la formación clásica», fundamentalmente en el «cómo se enseña», mediante la aplicación prioritaria del método de aprendizaje de formación inversa, la formación complementaria en el aula y/o la formación personalizada.
El desarrollo efectivo de este modelo de competencias se basa en un proceso de «reinvención de la formación clásica», fundamentalmente en el «cómo se enseña», mediante la aplicación prioritaria del método de aprendizaje de formación inversa, la formación complementaria en el aula y/o la formación personalizada

Una vez definidas las competencias y las habilidades del capital humano exponencial, se propone, a continuación, el marco formativo que las incorpora. 


\section{El modelo educativo inherente a las organizaciones expo- nenciales}

De acuerdo con lo expuesto, los modelos educativos inherentes a las organizaciones exponenciales necesitan incorporar unas competencias específicas, adaptadas a las necesidades de los modelos de estas singulares organizaciones.

Cabe señalar que la lógica de crecimiento exponencial contrasta con la lógica lineal en muchos ámbitos, pero, específicamente, en el educativo, donde lo habitual es aprender por acumulación de conocimientos y donde no se prevén saltos exponenciales. El motivo, tal y como se señala en Zoem Innovación Educativa (2018) y en Mana y De Giovanni (2018), es que las teorías que explican la educación actual, en su gran mayoría, nacen de la era industrial lineal que dominó gran parte del siglo XX, con un modelo de aprendizaje más basado en la tarea (Reigeluth, 2016).

Para continuar con la exposición del modelo educativo, se propone un marco formativo adaptado a las necesidades de las organizaciones exponenciales, a la innovación disruptiva y, más concretamente, al rasgo más significativo, el liderazgo disruptor, basado en la lógica de la abundancia, en el sentido expuesto por Diamandis (2016).

\subsection{Taxonomía competencial asociada al liderazgo disruptor}

Por lo que respecta a la búsqueda de la existencia de experiencias enmarcadas en la elaboración de taxonomías, se han encontrado dos ámbitos: liderazgo de equipos y liderazgo estratégico. En el ámbito de competencias del liderazgo de equipos asociadas a diseños de programas (Saiz, 2011) existen numerosos programas y métodos de entrenamiento que han confirmado su eficacia en diferentes colectivos de profesionales, como tripulaciones aéreas, unidades militares o equipos sanitarios.

Entre las técnicas más extendidas y contrastadas, destacan el entrenamiento cruzado (los miembros obtienen información sobre los roles de los demás), metacognitivo (se desarrollan habilidades que regulan procesos como razonamiento inductivo y deductivo y solución de problemas), la coordinación del equipo (orientado a que los miembros conozcan y manejen los procesos que determinan el trabajo en equipo efectivo), la autocorrección (los miembros aprenden habilidades para analizar su propio desempeño, revisar los hechos, intercambiar retroalimentación y planificar futuras actuaciones), la exposición a situaciones de estrés (los miembros aprenden los principales estresores que pueden perjudicar el desempeño del equipo y las estrategias de afrontamiento eficaces) y el desarrollo del equipo o teambuilding (orientado a mejorar el funcionamiento global del equipo centrándose en la clarificación de roles, en la fijación de metas, en la solución de problemas y en la mejora de las relaciones interpersonales) (Gil, Alcover, Rico y Sánchez-Manzanares, 2011). 
Respecto al ámbito del liderazgo estratégico, el caso específico de las Fuerzas Armadas de Canadá (Aznar, 2018) nos dice que el modelo de competencias se ha venido a convertir en la respuesta a los deseos prácticos de la sistematización. Las Fuerzas Armadas Canadienses, en esta línea y en un alarde académico de taxonomía de sumo interés, distinguen cinco elementos decisivos para definir el desarrollo personal que conduce al liderazgo estratégico y que muestra la versatilidad que demanda la evolución del militar profesional. Estos elementos son la experiencia, las capacidades cognitivas, las capacidades sociales, la capacidad de cambio y la ideología profesional. La tipología de estos elementos varía según el nivel considerado: júnior, intermedio, avanzado y sénior. Tras este análisis, comprobamos cómo la transformación digital crea nuevos desafíos para individuos y organizaciones, y todas esas nuevas habilidades necesarias están vinculadas a los fundamentos de la taxonomía de competencias de liderazgo disruptor.

Es en este punto cuando se plantea la identificación de las competencias asociadas al liderazgo disruptor. Con este fin, y en esta circunstancia, se han llevado a cabo 33 entrevistas en profundidad en el contexto del canal BeDigital -espacio de divulgación de las nuevas tecnologías aplicadas a la transformación digital, lo exponencial y la disrupción actuales(www.be-digital.es) (BeDigital, 2018); una serie de entrevistas realizadas a directivos y mandos intermedios, profesionales del mundo empresarial que están vinculados al contexto de la digitalización y a diferentes sectores de actividad. Los resultados de dicho análisis nos permiten afirmar que las principales competencias son las llamadas «competencias blandas" combinadas, junto a nuevos tipos de competencias inherentes a la estrategia digital y a la transformación digital de las empresas. Este contexto métrico nos permite conformar lo que denominamos «una taxonomía específica de competencias» relacionadas con el modelo de liderazgo disruptivo que mostramos en el cuadro 5.

Cuadro 5. Taxonomía competencial del liderazgo disruptor

\begin{tabular}{|c|c|c|}
\hline \multicolumn{3}{|c|}{ Tipo de competencias } \\
\hline $\begin{array}{l}\text { 1. }{ }^{a} \text { clase. Competencias } \\
\text { «estrategia digital» }\end{array}$ & $\begin{array}{l}\text { 2. }{ }^{a} \text { clase. Competencias } \\
\text { «blandas» }\end{array}$ & $\begin{array}{l}\text { 3. }{ }^{a} \text { clase. Competencias } \\
\text { «transformación digital» }\end{array}$ \\
\hline Evidencias & Evidencias & Evidencias \\
\hline Dirigir los procesos de transfor- & Comunicación. & Pensamiento crítico. \\
\hline mación digital. & Trabajo en equipo. & Comunicación compleja. \\
\hline Organización de visión holística. & Toma de decisiones. & Creatividad. \\
\hline Definir la estrategia basada en & Resolución del problema. & Colaboración. \\
\hline tecnología cliente-empresa. & Empoderamiento. & Flexibilidad y adaptabilidad. \\
\hline $\begin{array}{l}\text { Promover la cultura digital de las } \\
\text { personas. }\end{array}$ & Empatía. & Productividad y evaluación. \\
\hline $\begin{array}{l}\text { Capacidad de desarrollo de nue- } \\
\text { vos modelos digitales. }\end{array}$ & & \\
\hline
\end{tabular}




\section{Propuesta para construir una acción formativa exponencial}

Una vez definida la taxonomía competencial del liderazgo disruptor, y teniendo como base dicha premisa, será preciso establecer el ecosistema de aprendizaje y un nuevo marco formativo, adecuado a la adquisición de dichas competencias en el contexto de las organizaciones exponenciales. De esta forma, se logrará transferir la adquisición de dichas competencias dentro de un marco formativo específico, conducente al logro de los objetivos de aprendizaje propuestos y, al mismo tiempo, adaptado al sistema de aprendizaje denominado «aprendizaje invertido».

\subsection{El ecosistema de aprendizaje}

El nuevo marco formativo que se propone, aplicable al contexto de las organizaciones exponenciales, y que se ajusta al objetivo específico de nuestra propuesta investigadora es el denominado «aprendizaje invertido» (flipped learning model).

Tomando en cuenta la taxonomía de Staker y Horn (2012), así como el análisis realizado por Davies, Dean y Ball (2013) y Coufal (2014), el sustento teórico del aprendizaje invertido se puede forjar como se presenta en la figura 4.

Figura 4. ¿Cuál es el soporte teórico-pedagógico?

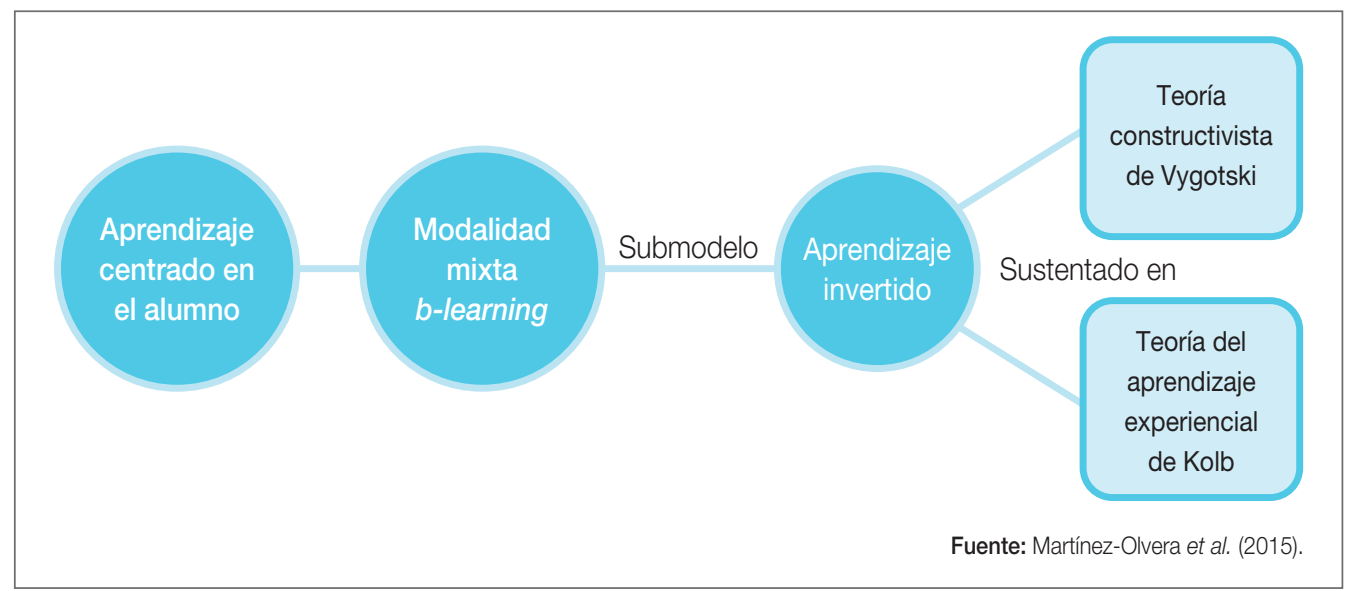

En este método se enseña y aprende por medio de la TIC, ofreciendo la posibilidad de enseñar instrucciones y materias diferenciadas. Además, de esta manera, los alumnos pueden estudiar un contenido determinado, adaptado a su propio ritmo, tantas veces como lo deseen, teniendo más autonomía y responsabilidad. 
Según Kanninen y Lindgren (2015), este método compromete la participación activa del alumno durante todo el curso de forma primordial y permite que el profesor ofrezca un tratamiento más individualizado a cada alumno. Cuando se realiza con éxito, abarca todas las fases del ciclo de aprendizaje según la taxonomía de Bloom (López, 2015). Tomando como base la experiencia de los profesores, las ventajas del método se notan más claramente en el caso de los alumnos más débiles, pero sirve también para reforzar la motivación de los alumnos más avanzados.

Es evidente que los alumnos universitarios son cada vez más «diginativos». Están acostumbrados a buscar información en formato digital y muchas veces prefieren estudiar fuera del aula tradicional.

Teniendo en cuenta lo mencionado previamente por Gómez y Muñoz (2004), es difícil identificar los mecanismos y comprender los sistemas por los que interactúan las actividades de aprendizaje y la creación de nuevos conocimientos para crear valor. Además, estas prácticas se desarrollan a lo largo de un periodo de tiempo muy prolongado e implican aspectos culturales, habilidades directivas y relaciones interpersonales difícilmente imitables. Una vez definido el ecosistema de aprendizaje y el soporte teórico-pedagógico, a continuación, se plantean los objetivos de aprendizaje propios del marco de aprendizaje propuesto.

\subsection{Objetivos de aprendizaje}

Siguiendo con la exposición de la propuesta del marco formativo, seguidamente, se acometerá la definición de los objetivos de aprendizaje propuestos, para abordar un diseño instruccional dentro del marco de este ecosistema de aprendizaje; un ecosistema caracterizado por un enfoque pedagógico, como el definido en Network (2014), donde la instrucción directa se desplaza de la dimensión del aprendizaje grupal a la dimensión del aprendizaje individual, transformándose el espacio grupal restante en un ambiente de aprendizaje dinámico e interactivo en el que el facilitador guía a los alumnos en la aplicación de los conceptos y en su involucración creativa con el contenido del curso. Por consiguiente, se entiende como el idóneo para abordar los planes de formación corporativos que favorezcan la adquisición de los tres tipos de competencias que totalizan el liderazgo disruptor.

Objetivo general de aprendizaje:

- La comprensión amplia del potencial de negocio y transformación de las tecnologías emergentes.

Objetivos específicos de aprendizaje:

- Reforzar la capacidad de elaborar visiones creativas que anticipen el futuro.

- Saber identificar las oportunidades que ofrece el entorno lo antes posible. 
- Ayudar a fortalecer las capacidades internas, al ser la estrategia mucho más volátil y difícilmente planificable, como variables fundamentales de la composición de la estrategia digital.

- Dar valor al impacto de la tecnología en las decisiones estratégicas.

- Aprender a identificar las tendencias tecnológicas (existencia y evolución de las tecnologías disruptivas) que puedan modificar los modelos de negocio.

- Conocer los criterios necesarios para modificar la dirección estratégica a la hora de reordenar los modelos de negocio.

\subsection{Características del diseño instruccional}

El modelo de diseño instruccional que se propone desarrollar en este tipo de ecosistema y marco-referencia de aprendizaje se cree que supondrá un cambio transcendental en el ámbito de la formación para el desarrollo del talento de las compañías disruptivas.

La finalidad de este cambio, basándose en la continua evolución de las metodologías de trabajo y en el brote incesante de nuevas herramientas y procesos tecnológicos, es estimular, conseguir e implantar el concepto «learnability» (término acuñado por Mara Swan como «la curiosidad y la capacidad de aprender nuevas habilidades para mantenerse empleable a largo plazo» (Sáenz, 2017).

Con este fin, el diseño instruccional propuesto presentará las características que a continuación se detallan:

- Sistema de enseñanza. Metodología educativa de aprendizaje invertido.

- Duración. Acciones formativas caracterizadas por la brevedad y el desarrollo en un horizonte temporal de corto plazo.

- Formato. Comunidades de aprendizaje.

- Modalidad. Híbrida -virtualización/presencial- (online y blended learning).

- Herramientas y procesos tecnológicos propuestos:

- Gamificación: implica los conceptos de «juego» y «diversión».

- Realidad aumentada: simulaciones de espacios reales en la virtualidad.

- Bibliotecas de recursos electrónicos: contenidos formativos y recursos bibliográficos complementarios. 
- Inteligencia artificial: análisis de datos con grandes variables basadas en la identificación:

- Habilidades requeridas actuales, previsibles y futuras.

- Conocimiento de sus intereses y experiencias.

- Como objetivo que se quiere alcanzar, se podrían ofrecer trabajos o responsabilidades de futuro con sentido para los empleados y reforzar el engagement en las empresas.

- Otro tipo de herramientas tecnológicas colaborativas internas y externas que permitan la obtención del conocimiento e información-formación actualizada.

- Sistema de evaluación. A través de la integración del indicador «taxonomía competencial del liderazgo disruptor» en el cuadro de mando de la estrategia digital de la organización.

\section{Conclusiones}

Como resultado del trabajo de investigación, se ha puesto de manifiesto que la sociedad y la economía se encuentran en una profunda transformación, apoyada en las TIC y en procesos profundos de digitalización, dentro del contexto de la nueva economía y de la innovación disruptiva.

El exponente de las organizaciones con mayores niveles de éxito son las organizaciones exponenciales. Se ha definido con detalle el modelo de negocio en el que se apoyan, así como las tecnologías disruptivas que están detrás de algunas de ellas.

Avanzando en el estudio, se establece la taxonomía competencial asociada al liderazgo disruptor como el conjunto de competencias identificadas y las más significativas, inherentes a la innovación disruptiva.

A la vista de lo anterior, se propone un marco formativo, capaz de responder a las competencias asociadas con el liderazgo y con la innovación disruptivas, estableciéndose, además, un marco de referencia completo con el aprendizaje centrado en el alumno.

Finalizando con el trabajo, y como consecuencia de todo lo anterior, corresponde en este punto establecer las conclusiones principales extraídas. Para comenzar con ellas, se plantea detallar, a continuación, en primer lugar, las futuras líneas de investigación. 


\subsection{Futuras líneas de investigación}

Por lo que se refiere a las futuras líneas de investigación del estudio, al contextualizarse dentro de unos conceptos nuevos y en constante cambio, como son la "transformación digital» y la «nueva economía», se plantean interesantes retos para futuros investigadores.

Como primera línea futura de investigación, puede ser de relevancia poner en práctica el marco formativo propuesto, medir los resultados y mejorarlo a la vista de la información obtenida. En segundo lugar, tendrá interés divulgar y difundir los resultados, con el posible objetivo de incorporar este marco de forma masiva en el sistema educativo y provocar así un cambio virtuoso en la educación.

Por último, se puede ampliar el marco formativo y generar nuevas líneas de investigación, al menos en dos ámbitos:

- Incorporando otros conjuntos de competencias más allá del liderazgo disruptor.

- Se podrá mejorar el marco de formación adaptándolo al previsible cambio que se provocará en el futuro en la nueva economía, las organizaciones exponenciales o las tecnologías disruptivas.

Una vez establecidas las futuras líneas de investigación asociadas al marco formativo, se exponen a continuación, las conclusiones principales.

\subsection{Conclusiones principales}

Las conclusiones del trabajo de investigación son las siguientes:

- En el contexto actual de la nueva economía y de la innovación disruptiva, los referentes son las organizaciones exponenciales, que incluyen competencias adicionales específicas. Con el objetivo de la adquisición de estas competencias, en esta investigación se propone un nuevo marco formativo, basado en la adquisición de las competencias inherentes al liderazgo disruptor, que mejora la competitividad de los profesionales y, por ende, de las empresas.

- El marco formativo propuesto en este trabajo mejora la competitividad de los profesionales y la competitividad empresarial de las organizaciones en el contexto de la nueva economía.

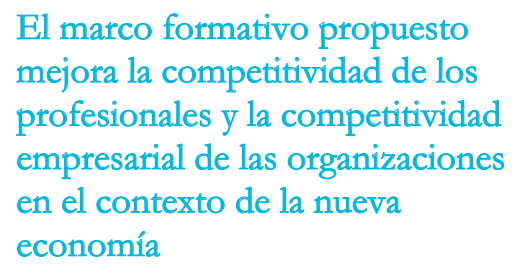

El marco formativo propuesto mejora la competitividad de los profesionales y la competitividad empresarial de las organizaciones en el contexto de la nueva economía 
- En conjunto, se ha diseñado un nuevo marco formativo que responde a la realidad de los nuevos profesionales, las nuevas profesiones, las que están y las que vendrán, que, además, podrá ser mejorado a medida que avanza la nueva economía. La aplicación práctica y exponencial del marco formativo permitirá aumentar la competitividad de la economía en su conjunto en el contexto de innovación disruptiva.

\section{Referencias bibliográficas}

Aguinis, H. y O'Boyle, E. (2014). Star performers in twenty-first century organizations. Personnel Psychology, 67(2), 313-350. doi: https://doi.org/10.1111/peps.12054.

Andrea, M., Jonathan, P., Sarah, H. y Halah, T. (2019). Global 2000-The World's Largest Public Companies 2019. Recuperado de <https://www.forbes.com/global2000/\#78 $7 \mathrm{e} 2 \mathrm{ec} 6335 \mathrm{~d}>$ (consultado el 15 de diciembre de 2019).

Australian Government. (2013). ICT Workforce Study. Recuperado de <https://nww.google. com/url?sa=t\&rct=j\&q=\&esrc=s\&source=web\& $c d=\& v e d=2 a h U K E$ wiH5eP83vLqAhV5AmMBH epFDb0QFjACegQIBXAB\&url=https\%3A\%2F\% 2Fapo.org.au\%2Fsites\%2Fdefault\%2Ffiles\%2F resource-files\%2F2013-07\%2Fapo-nid34886. pdf\&usg=AOvVaw3TaOLsfxyWfzD1jdjAS8Rc $>$ (consultado el 10 de noviembre de 2019).

AznarFernández-Montesinos, F. (2018). Repensando el liderazgo estratégico. Madrid, España: Sílex Universidad.

Be Digital. (2018). Be Digital-Una ventana a la transformación digital. Recuperado de <https://www.be-digital.es> (consultado el 1 de octubre de 2019).

Birkin, F., Polesie, T. y Lewis, L. (2009). A new business model for sustainable development: an exploratory study using the theory of constraints in nordic organizations. Business Strategy and the Environment, 18(5), 277-290. doi: https://doi.org/10.1002/bse.581.

Bloomberg, J. (2015). Innovation takes the exponential express. Wired. Recuperado de $<$ https://www.wired.com/insights/2015/01/ innovation-takes-the-exponential-express/> (consultado el 3 de octubre de 2019).

Bower, J. L. y Christensen, C. M. (1995). Disruptive technologies: catching the wave. Harvard Business Review. Recuperado de $<$ https://hbr.org/1995/01/disruptive-tech nologies-catching-the-wave> (consultado el 15 de marzo de 2018).

Briggs, B. y Shingles, M. (2015). Tech trends. The fusion of business and IT. Deloitte. SRC.

Bughin, J., Manyika, J., Woetze, J. y Labaye, E. (2016). Digital Europe: Pushing The Frontier, Capturing The Benefits. McKinsey \& Company.

Burke, B., Cearley, D., Jones, N., Smith, D., Chandrasekaran, A., Lu, C. K. y Panetta, K. (2019). Gartner top 10 strategic technology trends for 2020-Smarter with Gartner. Gartner, 52. Recuperado de <https://www.gartner.com/ smarterwithgartner/gartner-top-10-strategictechnology-trends-for-2020> (consultado el 5 de febrero de 2020). 
Comisión Europea. (2015). Una estrategia para el mercado único digital de Europa. Recuperado de <https://ec.europa.eu/digi tal-single-market/en/artificial-intelligence> (consultado el 6 de marzo de 2019).

Coufal, K. (2014). Flipped Learning Instructional Model: Perceptions of Video Delivery to Support Engagement in Eighth Grade Math. Beaumont, Texas, EE. UU.: Lamar University.

Davies, R. S., Dean, D. L. y Ball, N. (2013). Flipping the classroom and instructional technology integration in a college-level information systems spreadsheet course. Educational Technology Research and Development, 61(4), 563-580.

Diamandis, P. H. (2016). The road to abundance-Innovation, disruption, and opportunity. Research-Technology Management, 59(6), 20-24. doi: https://doi.org/10.1080/ 08956308.2016 .1232135$.

Dubinsky, Y. y Hazzan, O. (2019). Agile exponential softwareorganizations. Proceedings-2019 IEEE/ACM 41st International Conference on Software Engineering: Software Engineering in Practice, ICSE-SEIP 2019. doi. https://doi.org/10.1109/ICSE-SEIP.2019. 00027.

Farahani, S. S. S., Tourang, H., Yousefpour, B., Naraghi, M. G. y Javadian, S. A. M. (2012). Exponential modeling evaluation of time-of-use demand response programs in restructured power markets. Research Journal of Applied Sciences, Engineering and Technology, 4(1).

Gardner, H. (2012). La educación de la mente y el conocimiento de las disciplinas. Barcelona, España: Paidós.

Gil, F., Alcover, C. M.a , Rico, R. y SánchezManzanares, M. (2011). Nuevas formas de liderazgo en equipos de trabajo. Papeles del Psicólogo, 32(1), 38-47.
Gómez, F. G. y Muñoz, R. M. (2004). Las relaciones entre las actividades de aprendizaje y las nuevas tecnologías: su influencia en los resultados empresariales. Investigaciones Europeas de Dirección y Economía de la Empresa, 10(2), 93-113.

Ismail, S., Malone, M. S. y Van Geest, Y. (2014). Exponential Organizations: Why New Organizations Are Ten Times Better, Faster, and Cheaper Than Yours (and What to Do about It). Nueva York, EE. UU.: Diversion Books.

Kanninen, J. y Lindgren, K. (2015). ¿Por qué la clase invertida con TIC en la clase de ELE? Universidad de Turka (Finlandia). Centro Virtual Cervantes. Recuperado de <https:// cvc.cervantes.es/ensenanza/biblioteca ele/publicaciones_centros/PDF/estocol mo_2015/06_kaaninen-lindgren.pdf> (consultado el 25 de marzo de 2020).

Kim, W. y Mauborgne, R. (2014). Blue Ocean Strategy, Expanded Edition: How to Create Uncontested Market Space and Make the Competition Irrelevant. Boston, Massachusetts, EE. UU.: Harvard Business Review Press. Recuperado de <https://books.goo gle.com/books?hl=es\&lr=\&id=gwypBQAAQ BAJ\&oi=fnd\&pg =PR4\&dq =Kim, + W. + C.,$+\%$ 26+Mauborgne,+R.+(2005).+Blue+Ocean+ Strategy:+How+To+Create+Uncontested+ Market+Space+And+Make+The+Competiti on+Irrelevant,+Harvard+Business+Review+ Press.\&ots=bOb9LZca3s\&sig $=$ YCLCsPwHJ 33o-RZvRIfOxKNMewQ> (consultado el 23 de marzo de 2020).

Krüger, K. (2006). El concepto de sociedad del conocimiento. Revista Bibliográfica de Geografía y Ciencias Sociales, 11(683).

Kurzweil, R. (2004). The law of accelerating returns. En C. Teuscher (Ed.), Alan Turing: Life and Legacy of a Great Thinker (pp. 381416). Alemania: Springer-Verlag. doi: https:// doi.org/10.1007/978-3-662-05642-4_16.

Landeta, A. (2018). A Digital Transformation Strategy Model for Companies within the 
Industry 4.0 Framework (Tesis doctoral). MIU y Universidad Internacional de la Rioja, EE. UU./España.

Lee, Y., Nunes Amaral, L. A., Canning, D., Meyer, M. y Stanley, H. E. (1998). Universal features in the growth dynamics of complex organizations. Physical Review Letters, 81(15), 3.275-3.278. doi: https://doi.org/ 10.1103/PhysRevLett.81.3275.

López Moreno, M. (2015). ¿Qué es el aula invertida? Documento de internet. Nubemia. Recuperado de $<$ https://www.nubemia.com/aulainvertida-otra-forma-de-aprender/> (consultado el 27 de octubre de 2019).

Mana, F. y Giovanni, R. de. (2018). Claves para una educación exponencial (EdEx), 1-9. Recuperado de <https://www.google. $\mathrm{com} / \mathrm{url}$ ?sa $=\mathrm{t} \& \mathrm{rct}=\mathrm{j} \& \mathrm{q}=\& \mathrm{esrc}=\mathrm{s} \&$ source $=$ web\&cd=\&ved=2ahUKEwjs7_eGtKfqA hWCyoUKHV4VDNMQFjAAegQIARAB\&u $\mathrm{rl}=\mathrm{https} \% 3 \mathrm{~A} \% 2 \mathrm{~F} \% 2 \mathrm{Fencuentros}$.virtuale duca.red\%2Fstorage\%2Fponencias $\% 2 \mathrm{Fa}$ rgentina2018\%2Fh7Lwq6tizN2zFBmaYd8 hCshutYz4DDDn1b7aRsca.pdf\&usg=AOvV aw2DGFYbLznKnHQL17gaPH56> (consultado el 23 de abril de 2020).

Manyika, J., Chui, M., Bughin, J., Dobbs, R., Bisson, P. y Marrs, A. (2013). Disruptive Technologies: Advances that Will Transform Life, Business, and the Global Economy. San Francisco, California, EE. UU.: McKinsey Global Institute.

Martínez-Olvera, W., Esquivel-Gámez, I. y Martínez, J. (2015). Acercamiento teóricopráctico al modelo de aprendizaje invertido. Alternativas para Nuevas Prácticas Educativas, 1, 158-172.

Mayer, C. (2016). Sustaining innovation in the midst of success. Journal of Leadership Studies, 10(1), 73-75. doi. https://doi. org/10.1002/jls.21453.

Mohout, O. (2017). A Critical Perspective to Exponential Organizations and its Hyper Scalability, 1-11. Recuperado de <http://
mixel.be/files/pdf/Critical-to-exponential_ preprint.pdf> (consultado el 2 de febrero de 2020).

Negreiro, M. y Madiega, T. (2019). Digital Transformation. European Parliamentary Research Service.

Network, F. L. (2014). FLIP learning. ¿Qué es el aprendizaje invertido o flipped learning? (Traducción de R. Moreno). Recuperado de <http://www.flippedlearning.org/defin tion> (consultado el 15 de febrero de 2020).

Norlean. (2019). Tendencias tecnológicas en las empresas para 2020. Recuperado de $<$ https://norlean.com/blog/tendencias-tec nologicas-en-las-empresas-para-2020/> (consultado el 2 de junio de 2020).

Osterwalder, A. y Pigneur, Y. (2010). Business Model Generation: A Handbook for Visionaries, Game Changers, and Challengers. Hoboken, Nueva Jersey, EE. UU.: John Wiley \& Sons. Recuperado de <https:// books.google.es/books?hl=es\&lr=\&id=Uz uTAwAAQBAJ\&oi=fnd\&pg=PA9\&dq=Bus iness+Model+Generation:+A+handbook+ for+visionaries, +game+changers+and+ch allengers+Publisher:+John+Wiley+and+S ons, +Inc.,+Hoboken,+New+Jersey+(201 0)+Authors:+Alexander+Osterwalder+a> (consultado el 15 de octubre de 2019).

Palao Gil, F. J. (2016). La cuarta revolución industrial y las organizaciones exponenciales: ¿por qué estamos en la cuarta revolución industrial? La transformación digital ya no es suficiente. Executive Excellence: La Revista de Liderazgo, la Gestión y la Toma de Decisiones, 132, 36-38. Recuperado de <https://dialnet.unirioja.es/ejemplar/49 2839>.

Panetta, K. (2019). Contributor Kasey Panetta: Gartner top 10 strategic technology trends for 2020-smarter with Gartner. Gartner. Recuperado de <https://www.gartner.com/ 
smarterwithgartner/gartner-top-10-strategictechnology-trends-for-2020> (consultado el 15 de marzo de 2020).

Parlamento Europeo. (2019). Una agenda digital para Europa. Fichas Temáticas sobre la Unión Europa. Recuperado de <http:// www.europarl.europa.eu/factsheets/es/ sheet/64/una-agenda-digital-para-europa> (consultado el 12 de febrero de 2020).

Pitcher, G. (2017). Securing the future. New Electronics, 50(20), 20-22. doi: https://doi. org/10.4324/9781315389165-3.

Puckett, J., Boutenko, V., Hoteit, L., Polunin, K., Perapechka, S., Stepanenko, A. y Bikkulova, G. (2020). Fixing the global skills mismatch. BCG. Recuperado de <https:// www.bcg.com/publications/2020/fixingglobal-skills-mismatch.aspx $>$ (consultado el 7 de junio de 2020).

Reigeluth, C. M. (2016). Teoría instruccional y tecnología para el nuevo paradigma de la educación. Revista de Educación a Distancia (RED), 50. doi: https://doi.org/10.6018/ $\mathrm{red} / 50 / 1 \mathrm{a}$.

Sáenz Blanco, M. ${ }^{a}$ T. (2017). Mara Swan, vicepresidenta ejecutiva de Estrategia Global y Talento. 7 pasos para lograr la inclusión consciente. Observatorio de Recursos Humanos y Relaciones Laborales, 124, 44-51. Recuperado de <https://dialnet.uni rioja.es/servlet/articulo?codigo $=6027314>$ (consultado el 9 de junio de 2020).
Saiz, M. G. (2011). Una revisión constructiva de la gestión por competencias. Anales de Psicología/Annals of Psychology, 27(2), 473-497.

Sánchez, J. (2020). Los 9 principios de la transformación disruptiva. Mujeres Consejeras.com. Recuperado de <http://www. mujeresconsejeras.com/los-9-principiosde-la-transformacion-disruptiva/> (consultado el 2 de junio de 2020).

Solomon, L. K. y Pijl, P. van der. (2017). How to make an exponential business model to $10 \mathrm{X}$ growth. Singularity Hub. Recuperado de <https://singularityhub.com/2017/04/03/ how-to-make-an-exponential-businessmodel-to-10x-growth/amp/> (consultado el 15 de marzo de 2020).

Staker, H. y Horn, M. B. (2012). Classifying $\mathrm{K}-12$ blended learning. Innosight Institute.

Zazzerini, G. (2016). Markets and Strategies. The Exponential Organizations. Recuperado de <https://www.google.com/url?sa=t\&rct $=j \& q=\& e s r c=s \&$ source $=$ web $\& c d=\& v e d=2 a$ hUKEwilhNnlvqfqAhVUhlwKHTZhB5EQFjA AegQIBBAB\&url=https\%3A\%2F\%2Fpdfs. semanticscholar.org\%2F1732\%2F2b402e c99b584dd8fb64b9802c1a4ab27142.pdf \&usg=AOvVaw31sw9KtDRBwmcwmVMg GSEZ> (consultado el 3 de marzo de 2020).

Zoem Innovación Educativa. (2018). Claves para una educación exponencial EdEx. Recuperado de <https://issuu.com/fm1979/docs/edex> (consultado el 15 de enero de 2020). 


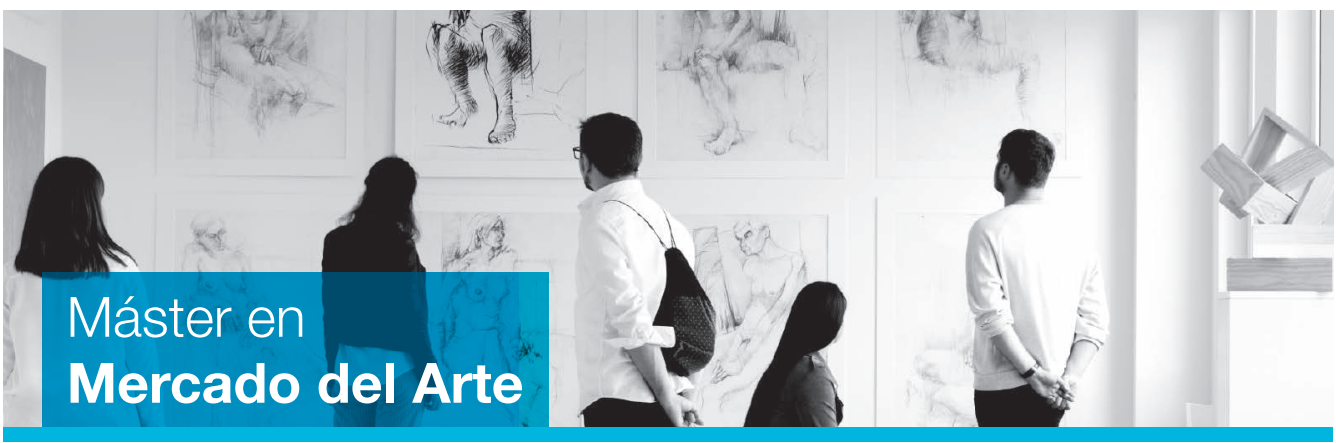

Este máster oficial de Mercado del Arte [60 créditos ECTS] tiene una duración normal de 12 meses.

Dirigido a: Titulados universitarios con un perfil amplio y diverso, que va desde el alumno con sesgo claramente humanista (como licenciados en Historia, Humanidades, Historia del Arte, Arqueología, etc.), hasta el alumno formado en finanzas, empresa y asesoramiento financiero que quiere encauzar sus conocimientos y experiencia hacia el mercado del arte y en el asesoramiento patrimonial en este tipo de activos. Tienen acceso directo al máster los titulados universitarios en Ciencias Empresariales, Administración y Dirección de Empresas y Economía. Aquellos titulados que no provengan de las titulaciones antes citadas deberán realizar unos complementos formativos.

Objetivos: Formar profesionales altamente cualificados en el ámbito de la dirección y gestión de empresas culturales relacionadas con el sector, dotándolos de un perfil práctico, profesional y competitivo. El programa formativo de este máster ha sido diseñado para que el estudiante domine todos los conocimientos necesarios que le capaciten para realizar adecuadamente y con garantía de éxito una labor de gestión, asesoramiento, peritaje y catalogación de bienes artísticos y de colección en entornos nacionales e internacionales.

\section{Publicaciones de interés}

\section{Área de Tecnología, Ciencia y Educación}

\section{Global e-learning}

Ana Landeta Etxeberria

(Coord.)

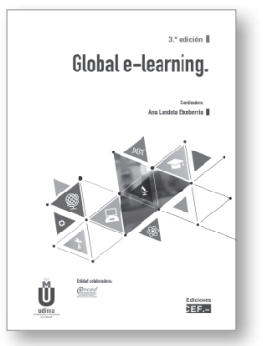

Esta obra analiza las principales tendencias actuales en el marco de la aplicabilidad de las TIC en diferentes escenarios educativos, así como 20 tecnologías emergentes y varias buenas prácticas asociadas a las mismas con un potencial tecnológico-pedagógico en el corto y medio plazo. Presenta 25 actividades didácticas innovadoras documentadas, realizadas por profesores expertos en el diseño instruccional más novedoso, y 23 proyectos europeos financiados principalmente por la Agencia Ejecutiva de Educación, Audiovisual y Cultura de la Comisión Europea (Education, Audiovisual and Culture Executive Agency [EACEA]) en el marco del programa Erasmus+ que aborda temáticas relacionadas principalmente con la innovación tecnológico-pedagógica.

En esta publicación, como en las anteriores ediciones del estudio internacional de e-learning que se lleva a cabo en la UDIMA desde hace varios años, han participado más de 166 instituciones y 56 autores procedentes de 32 países. 